\title{
Integrated Structural Analysis of N-glycans and Free Oligosaccharides Allows for a Quantitative Evaluation of ER Stress.
}

Naoki Fujitani ${ }^{1 *}$, Shigeru Ariki ${ }^{1,2}$, Yoshihiro Hasegawa ${ }^{1,3}$, Yasuaki Uehara ${ }^{1,3}$, Atsushi Saito $^{3}$, and Motoko Takahashi ${ }^{1}$

1 Department of Biochemistry, Sapporo Medical University School of Medicine, Sapporo 060-8556, Japan.

2 Department of Chemistry, Sapporo Medical University Center for Medical Education, Sapporo 060-8556, Japan.

3 Department of Respiratory Medicine and Allergology, Sapporo Medical University School of Medicine, Sapporo 060-8543, Japan.

* To whom correspondence should be addressed: Naoki Fujitani, Tel: +81-11-611-2111; e-mail: fujitani@sapmed.ac.jp

Table of contents:

- Figure S1. Schematic of the N-glycan biosynthetic pathway, N-glycosylated protein folding pathway, and $\mathrm{N}$-glycan degradation pathway conserved in eukaryotic cells.

- Figure S2. Representative MALDI-TOF MS spectra of N-glycans.

- Figure S3. Representative MALDI-TOF MS spectra of fOSs.

- Figure S4. Changes in fucosylation in hybrid/complex-type glycans in response to ER stress.

- Figure S5. Summary of N-glycome and fOS profile of HeLa cells treated with DTT.

- Figure S6. HCAs with heat map displaying the correlation between each ER stress-induced cell and the amount of glycans.

- Figure S7. HCA with heat map displaying the correlation between all ER stress-induced cells and the amount of glycans.

- Table S1. Summary of the estimated structure and quantitative values of all observed N-glycans in TM- and TG-treated cells.

- Table S2. Summary of the estimated structure and quantitative values of all observed fOSs in TMand TG-treated cells.

- Table S3. Summary of the estimated structure and quantitative values of all observed N-glycans in DTT-treated cells.

- Table S4. Summary of the estimated structure and quantitative values of all observed fOSs in DTTtreated cells. 


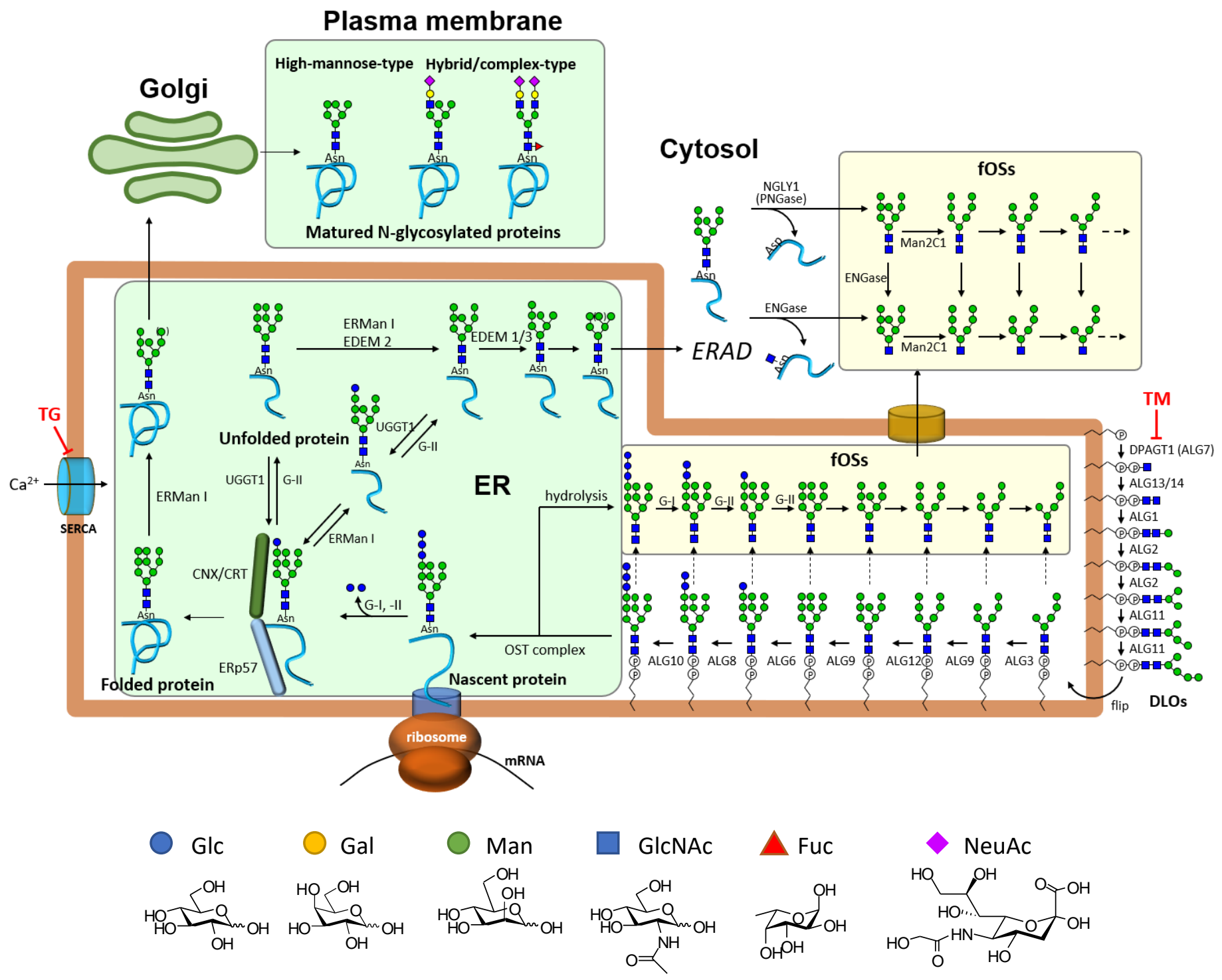

Figure S1. Schematic of the pathways of N-glycan biosynthesis, N-glycosylated protein folding, and $\mathrm{N}$-glycan degradation, which are conserved in eukaryotic cells. N-glycans on proteins and fOSs enclosed with green and yellow squares, respectively, are the analysis targets in this study. ER stress inducers, tunicamycin (TM) and thapsigargin (TG) inhibit DPAGT 1 at initial point of $\mathrm{N}$-glycan synthesis and $\mathrm{Ca}^{2+}$ transporter called SERCA on ER membrane, respectively, as indicated by red. 
N-glycans $(4 \mu \mathrm{g} / \mathrm{mL}$ TM-treated)

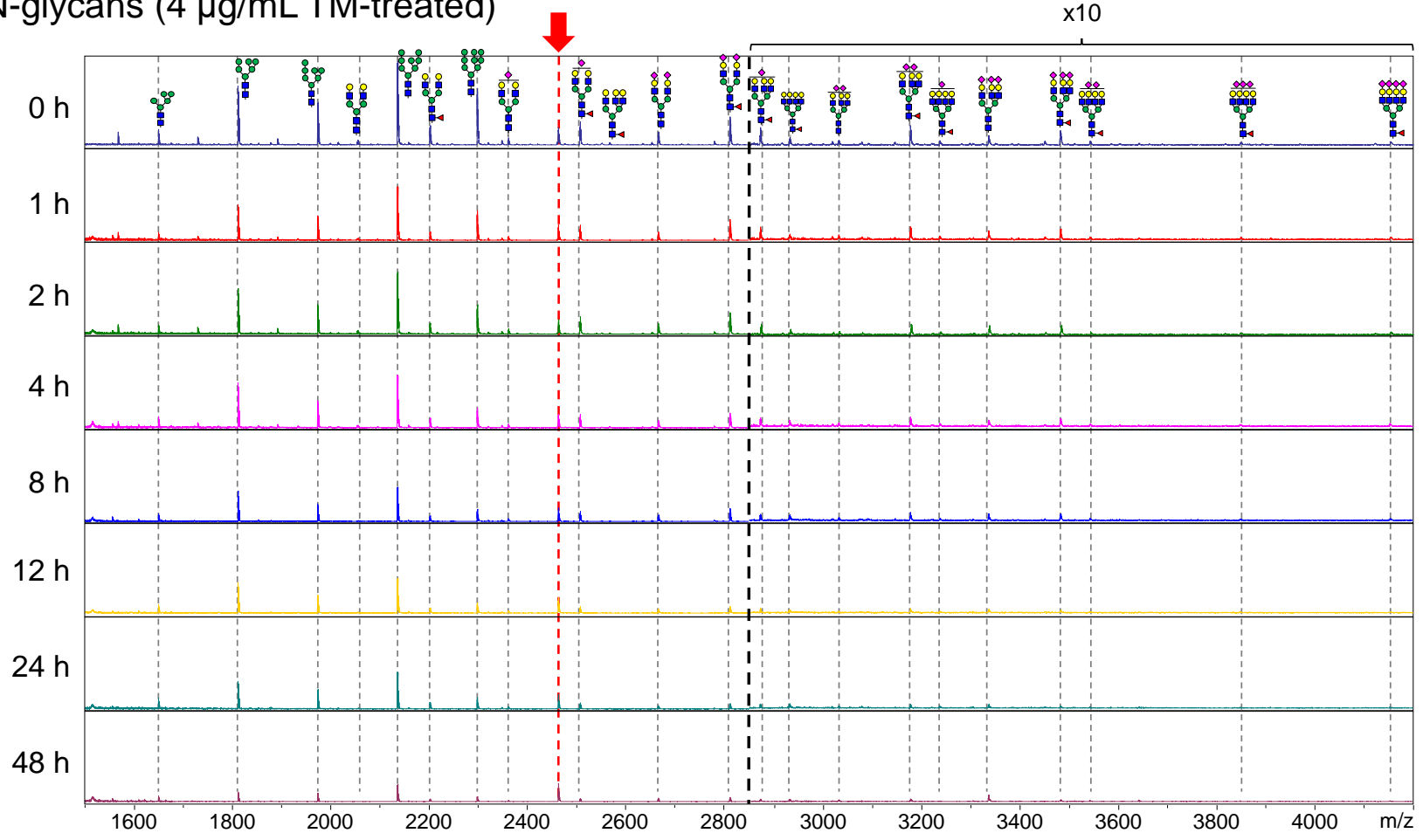

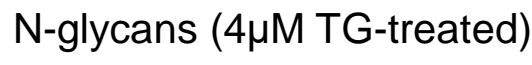

$\mathrm{x} 10$

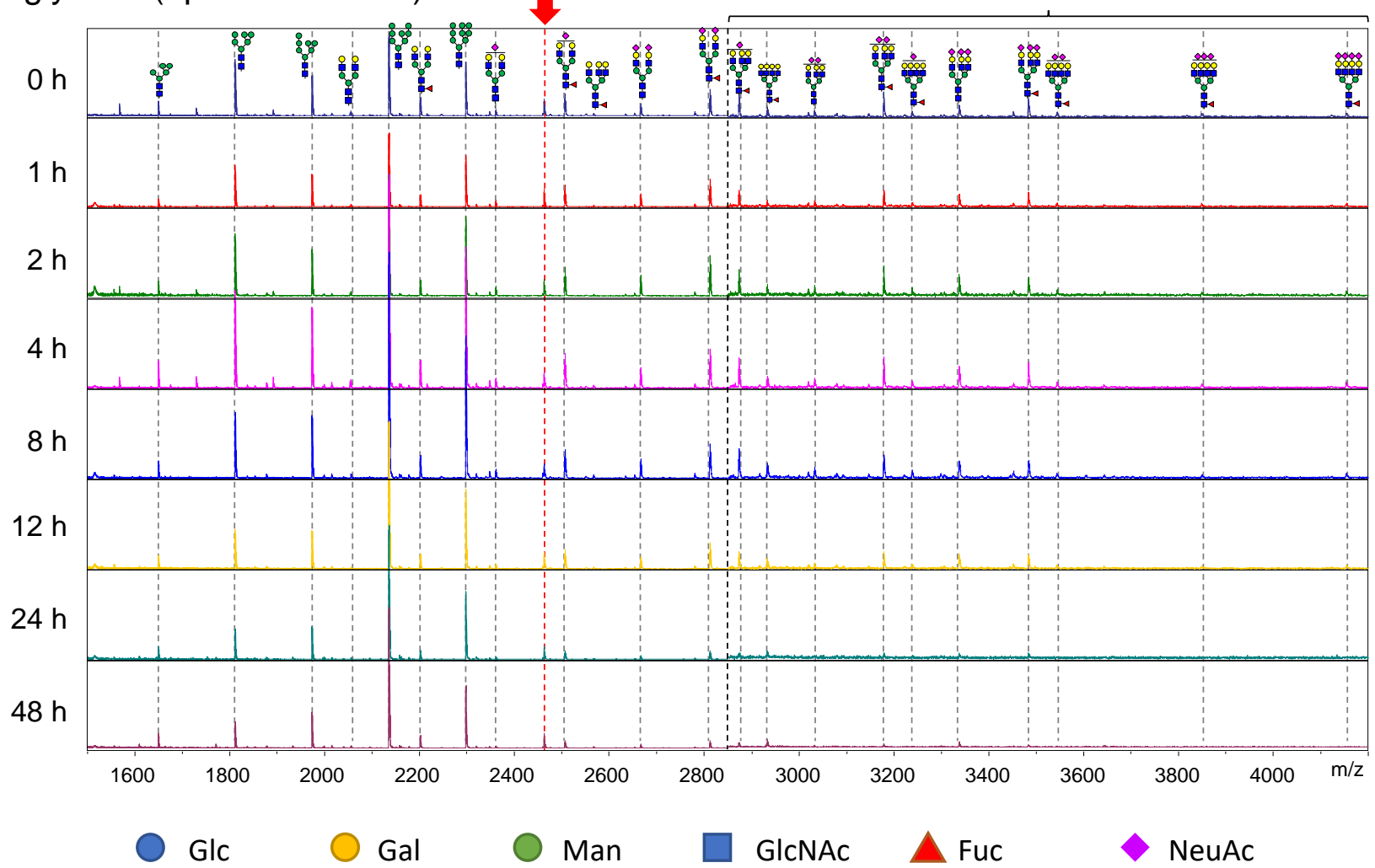

Figure S2. Representative MALDI-TOF MS spectra of N-glycans derived from TM- or TG-treated HeLa cells. All spectra were normalized to the area of I.S. (50 pmol) indicated by red dotted lines and arrows. The estimated structures were labeled to the major peaks. Region of $\mathrm{m} / \mathrm{z}$ more than 2850 is displayed at a $\times 10$ magnification. Each displayed spectrum was obtained from the cells treated independently with each inhibitor. 
fOSs (4 $\mu \mathrm{g} / \mathrm{mL}$ TM-treated)

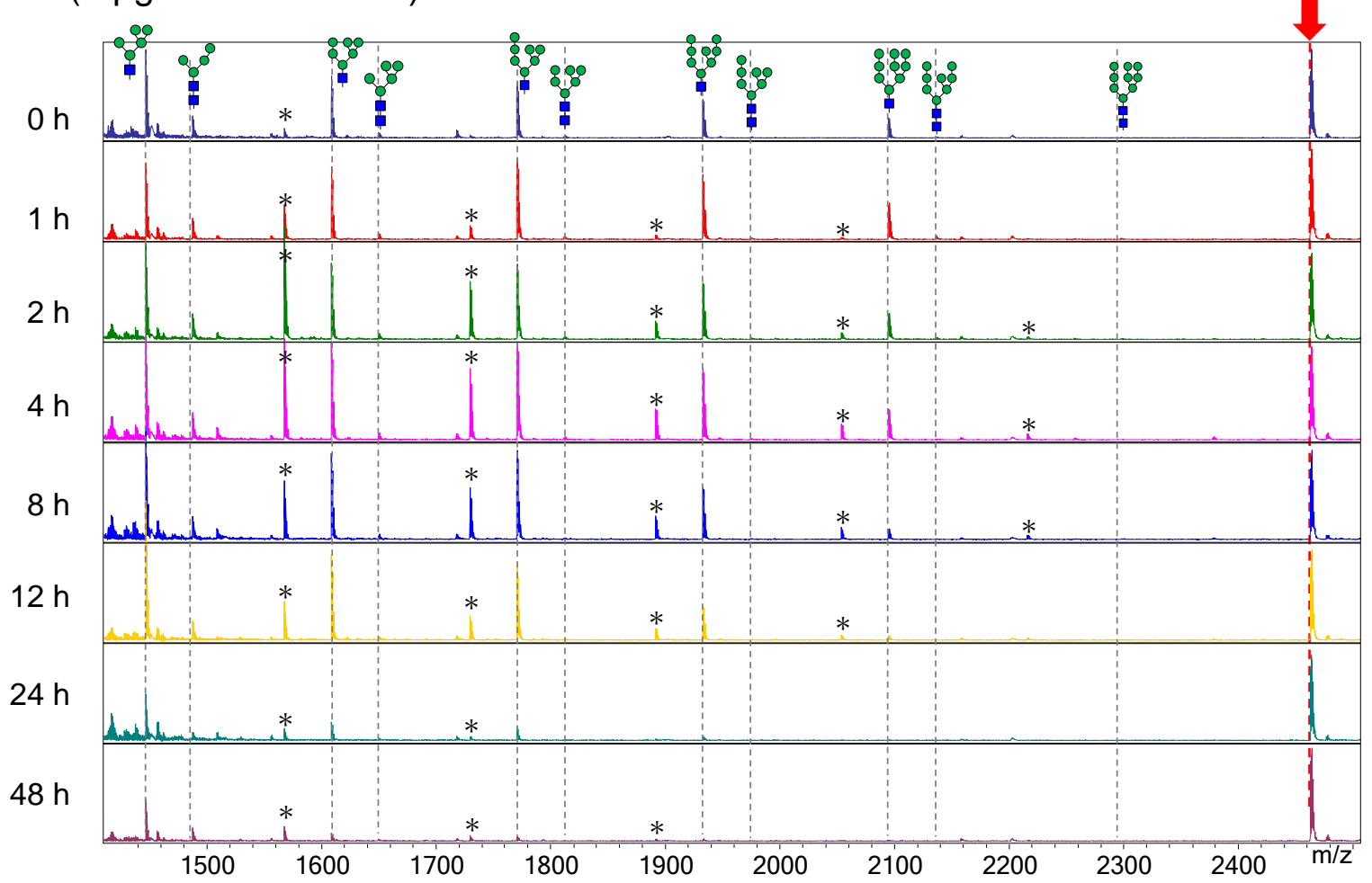

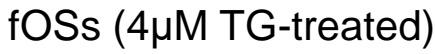

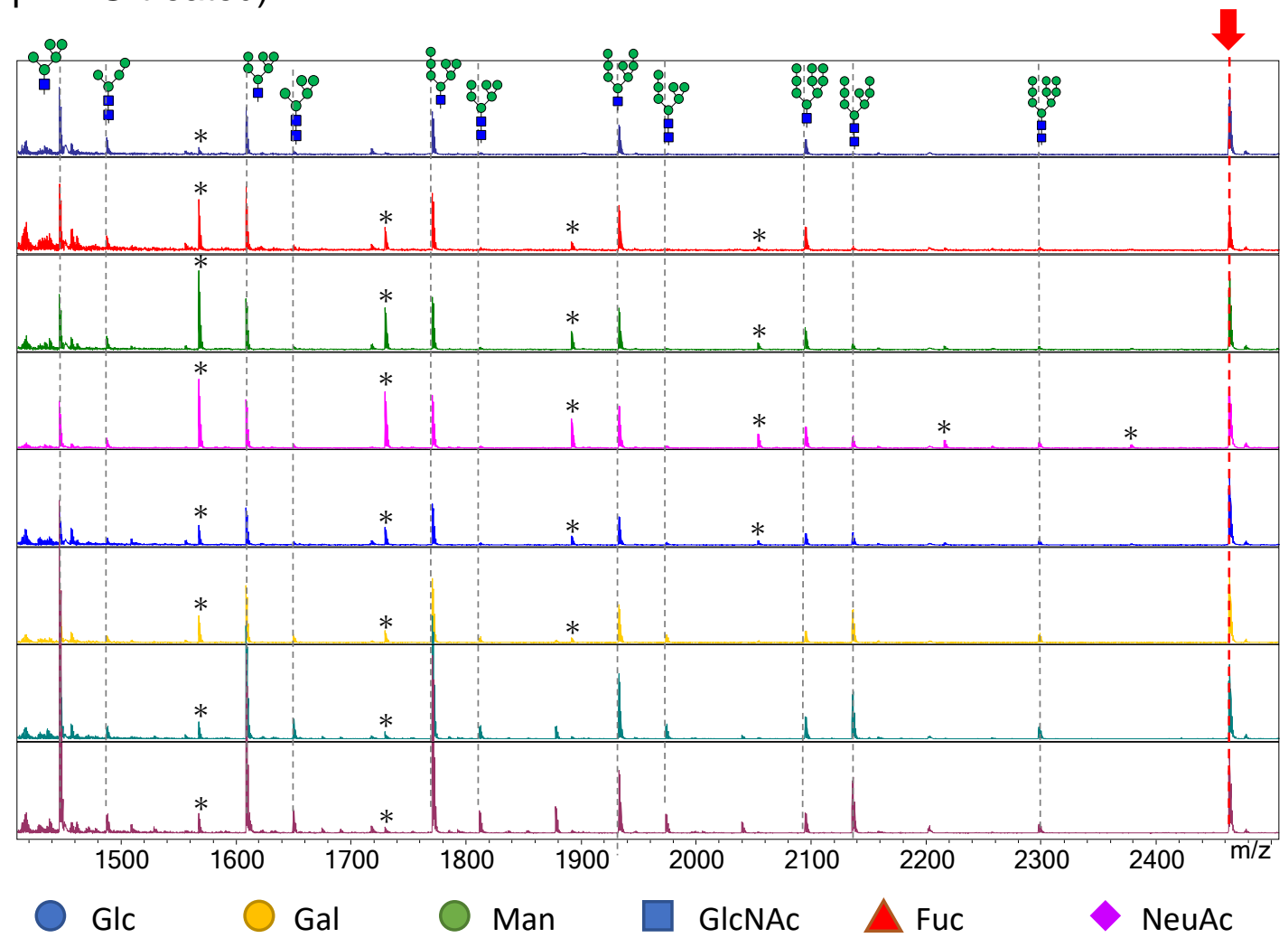

Figure S3. Representative MALDI-TOF MS spectra of fOSs derived from TM or TG treated HeLa cells. All spectra were normalized to the area of I.S. $(50 \mathrm{pmol})$ indicated by red dotted line. The estimated structures were labeled to the major peaks. The peaks labeled with asterisk (*) are hexose oligomers $(\mathrm{Hex})_{\mathrm{n}}$ from unknown origin. Each displayed spectrum was obtained from the cells treated independently with each inhibitor. 
A
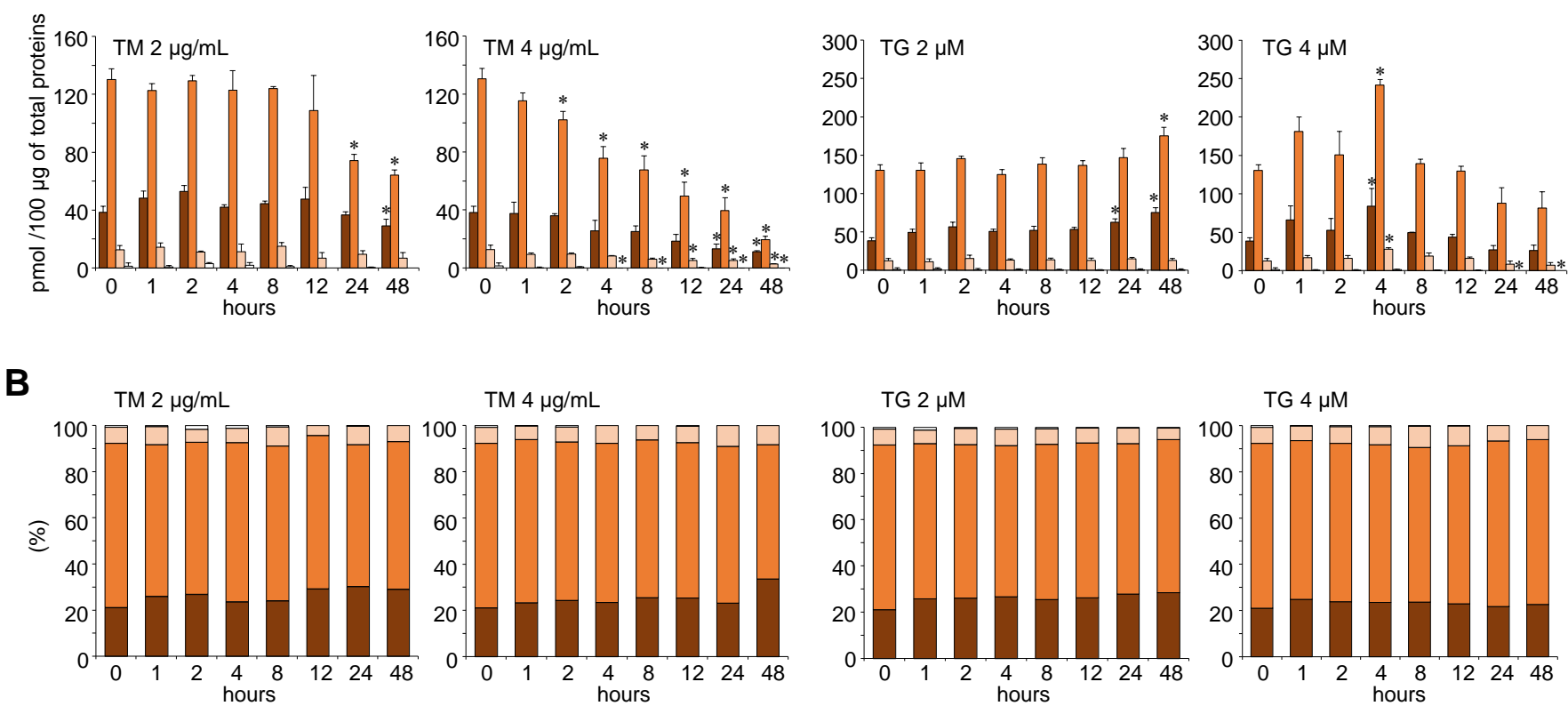

$\square$ Fuc x0 $\square$ Fuc x1 $\square$ Fuc x2 $\square$ Fuc x3

Figure S4. Changes in fucosylation in hybrid/complex type glycans in response to ER stress. Absolute (A) and relative (B) quantification of afucosylated (Fuc $\times 0$ ), monofucosyl (Fuc $\times 1$ ), difucosyl $($ Fuc $\times 2$ ), and trifucosyl (Fuc $\times 3$ ) hybrid/complex type glycans. Quantitative values are the mean + S.D. of three trials using cells treated independently with each inhibitor. Asterisks indicate a significant decrease or increase in amount compared to control cells ( $\mathrm{P}$ $<005)$. 


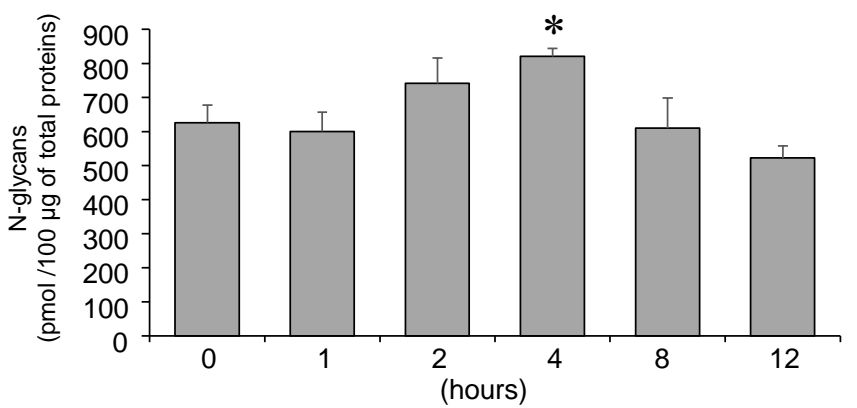

C

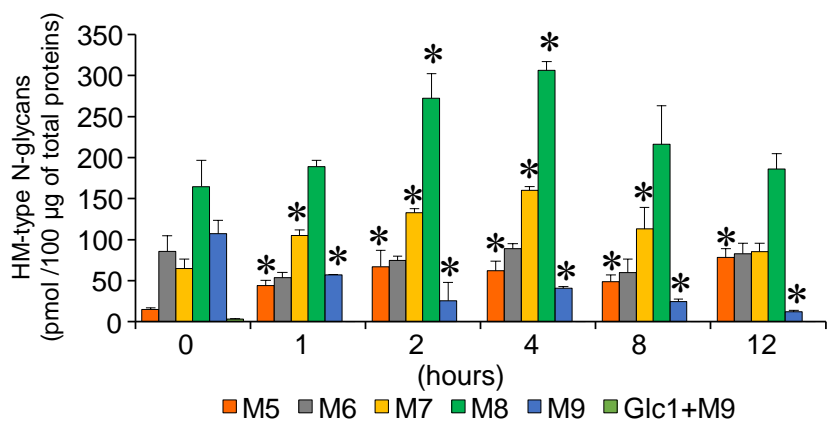

E

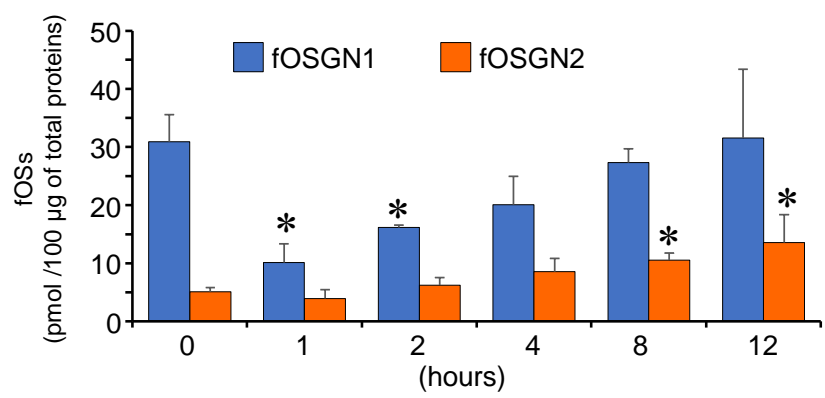

B

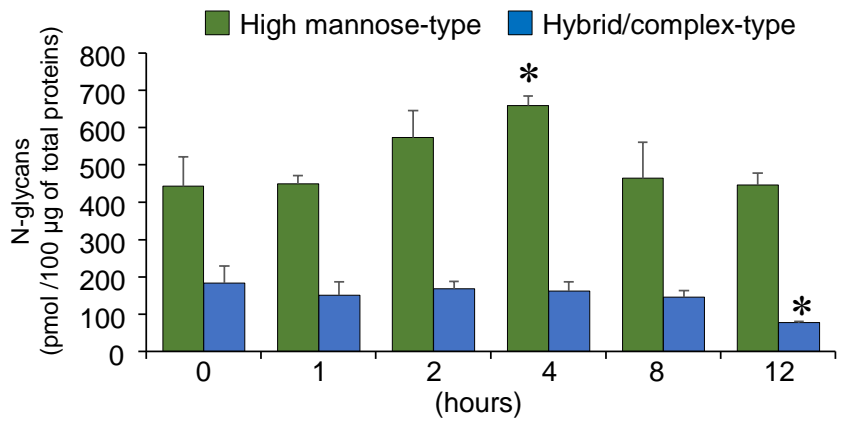

D

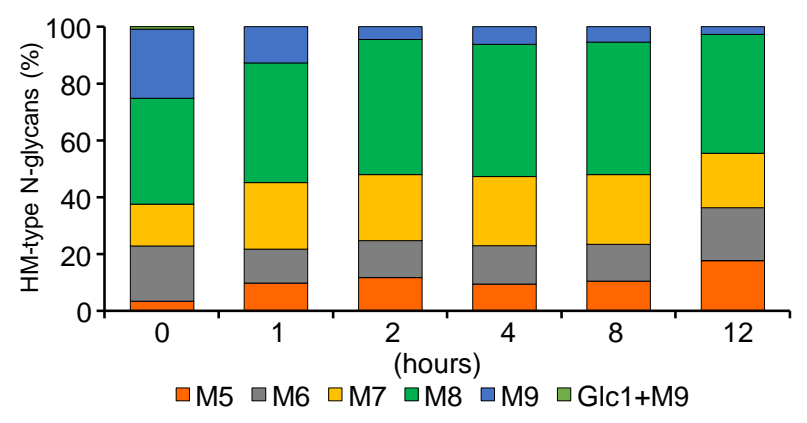

$\mathbf{F}$

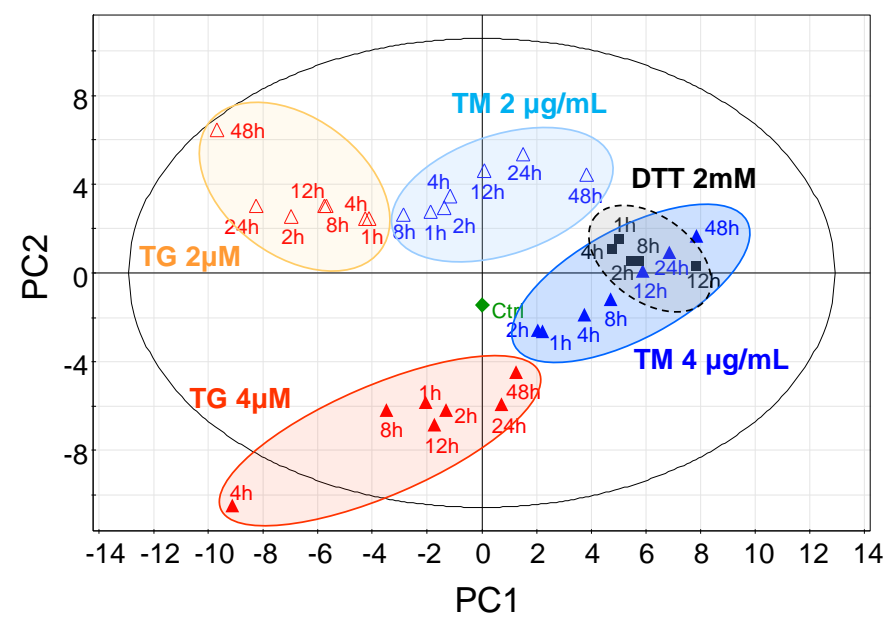

Figure S5. Summary of glycomic profile of $1.5 \mathrm{mM}$ DTT-treated cells. (A) Changes in the total amount of N-glycans. (B) Analysis of N-glycans by structural class (HM-type or Hybrid/complex-type). (C and D) Absolute (C) and relative (D) quantification of each structure of HM-type N-glycans. (E) Analysis of fOSs by structural class (fOSGN1 or fOSGN2). (F) PCA applying all glycomic data from TM- ,TG- and DTT-treated cells. The black line eclipse indicates the 95\% confidence of the Hotelling's T2 test. Quantitative values are the mean + S.D. of three trials using cells treated independently with each inhibitor. Asterisks indicate a significant decrease or increase in amount compared to control cells $(\mathrm{P}<005)$. 
$\square \begin{aligned} & 0.00 \\ & 3.33 \\ & 6.67 \\ & >10.00\end{aligned}$

(pmol/100 $\mu \mathrm{g}$ cellular proteins)

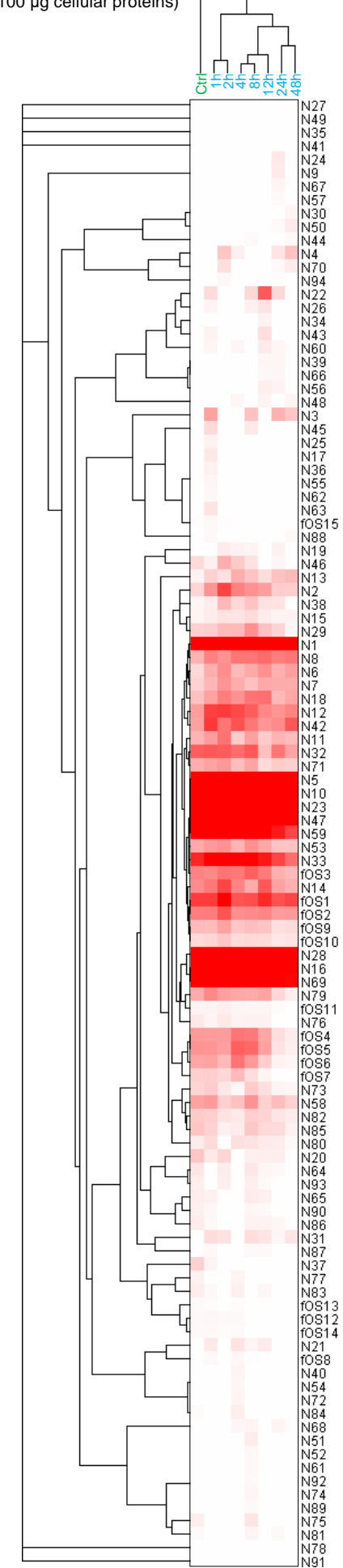

$2 \mu \mathrm{g} / \mathrm{mL}$ TM-treated

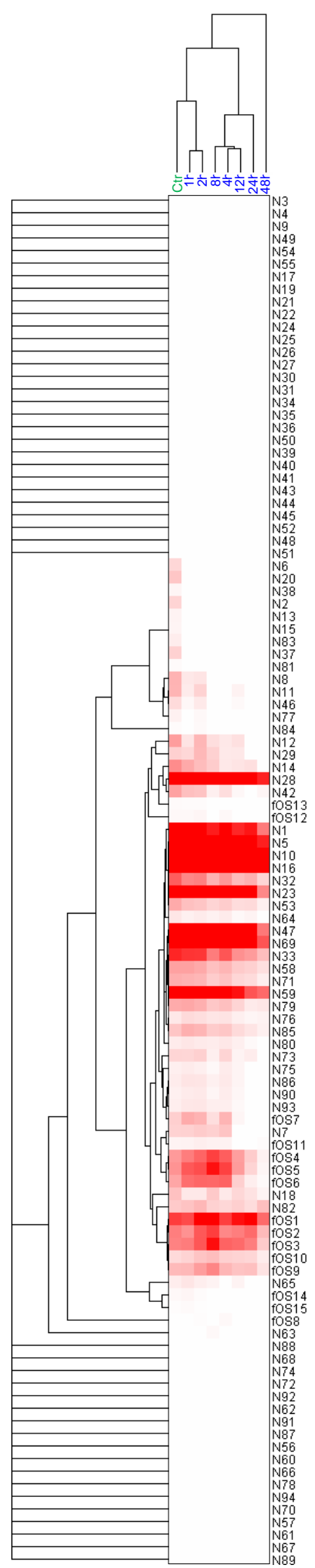

$4 \mu \mathrm{g} / \mathrm{mL}$ TM-treated

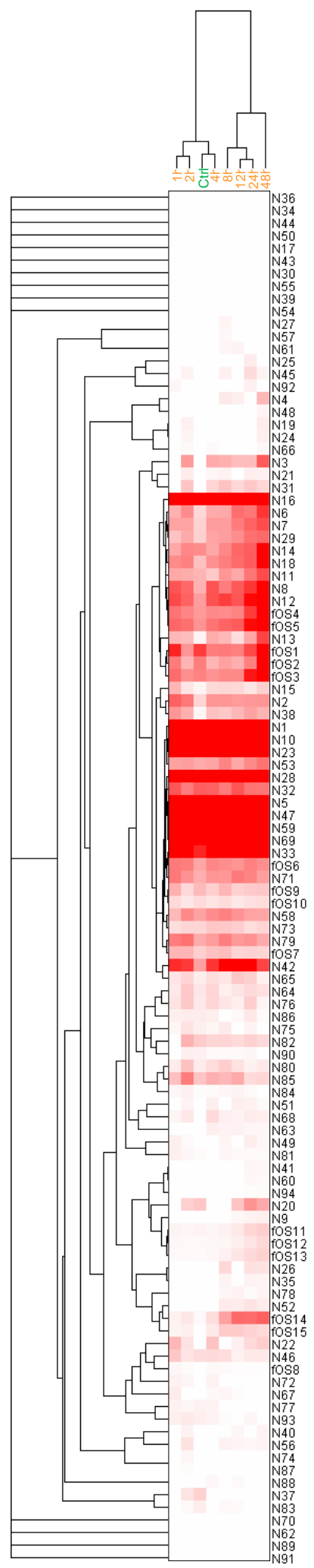

$2 \mu \mathrm{M}$ TG-treated

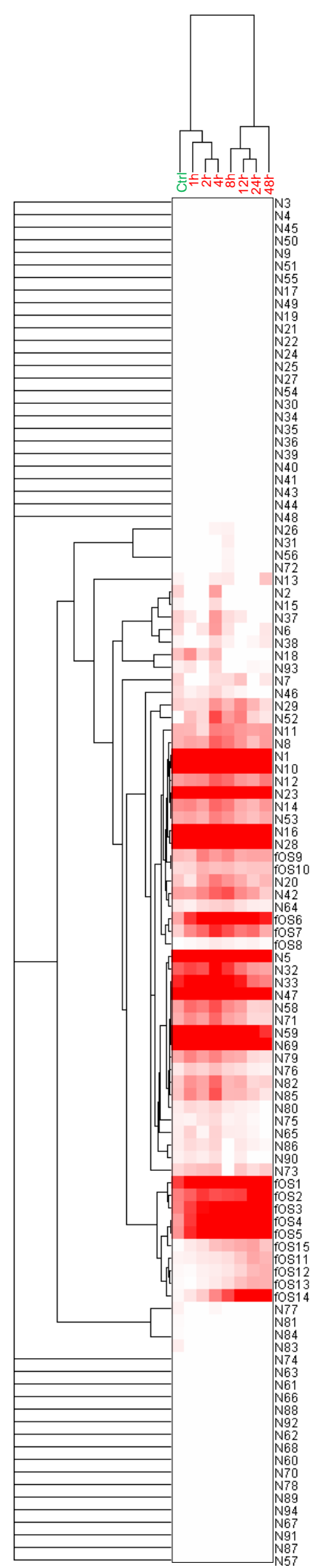

$4 \mu \mathrm{M}$ TG-treated

Figure S6. HCAs with heat map displaying the correlation between each ER stress induced cell and the amount of glycans. Notated glycan names refer to Tables S1 and S2. 


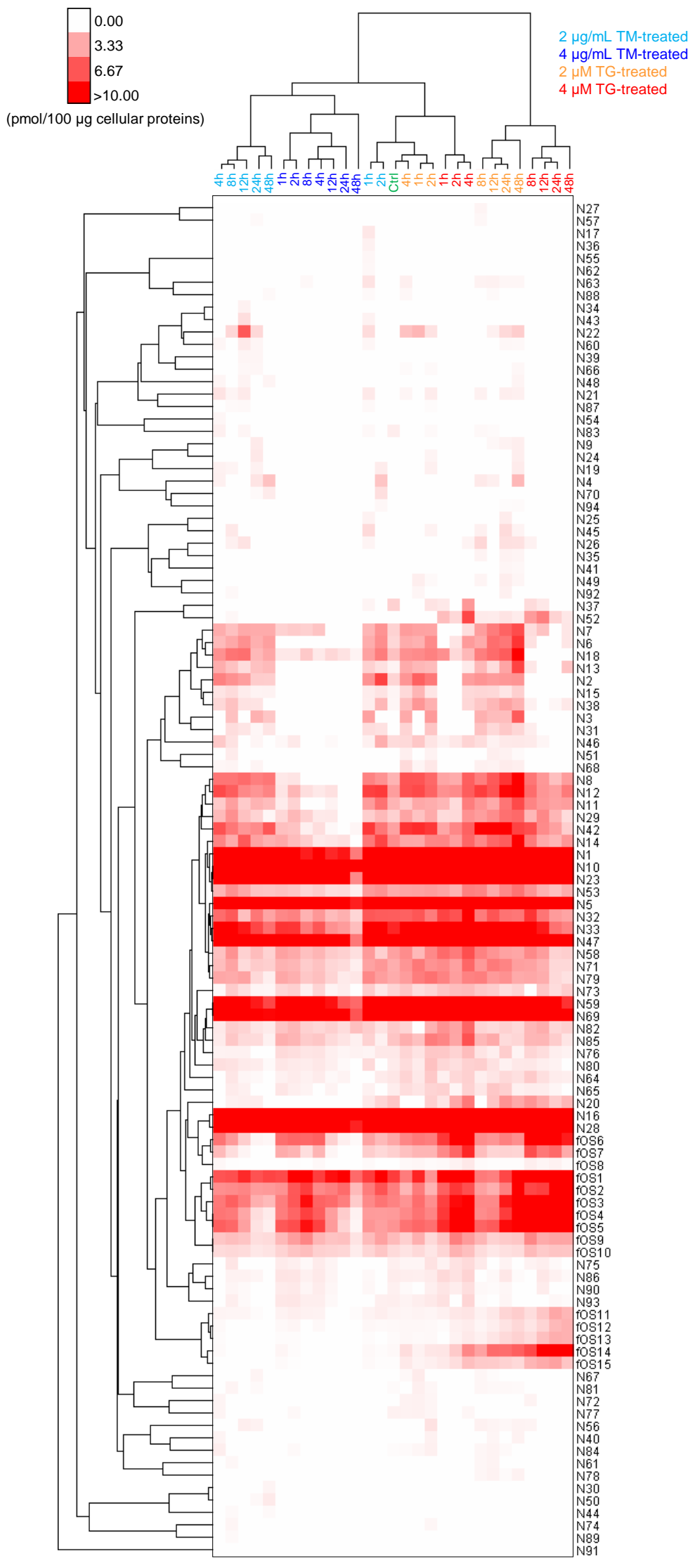

Figure S7. HCA with heat map displaying the correlation between all ER stress induced cells and the amount of glycans Notated glycan names are referred in Tables S1 and S2. 


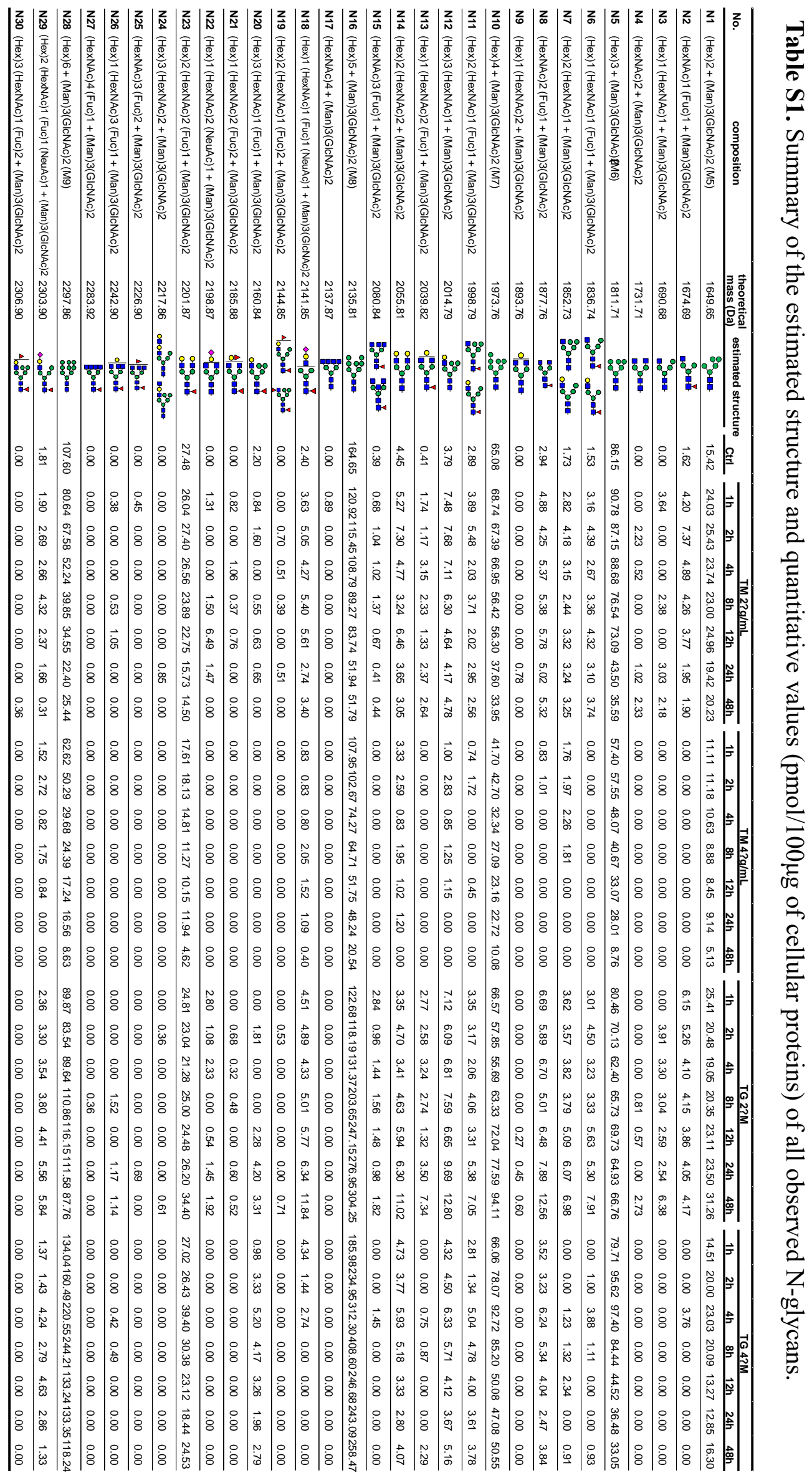




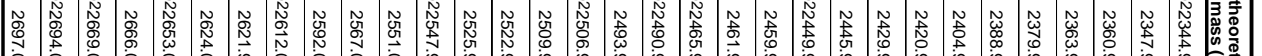

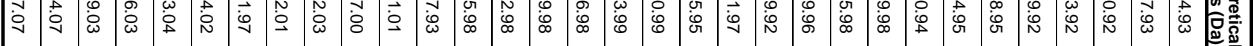

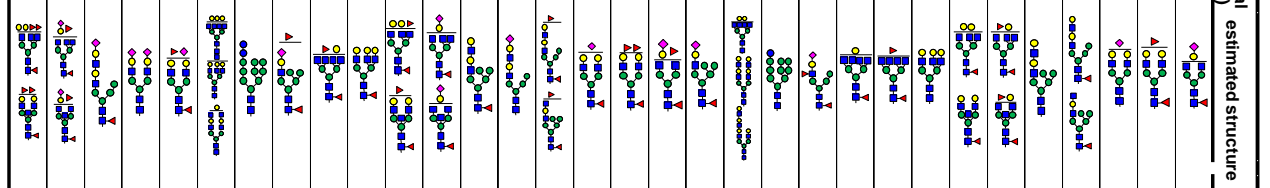

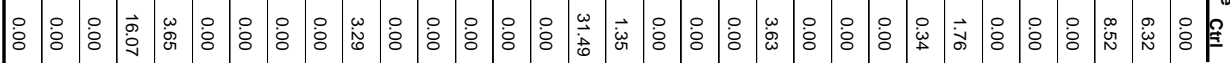

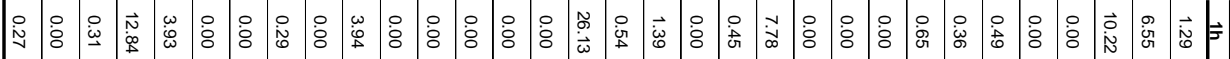

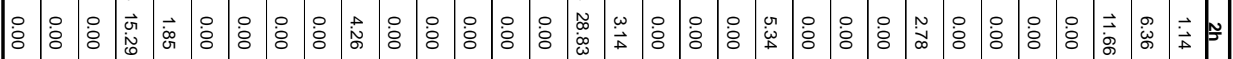

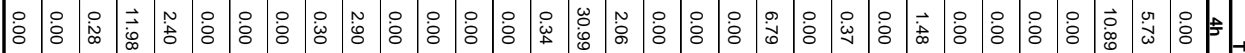

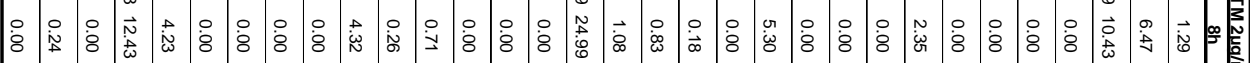

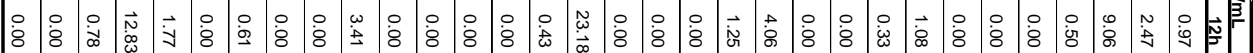

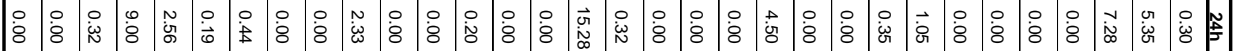

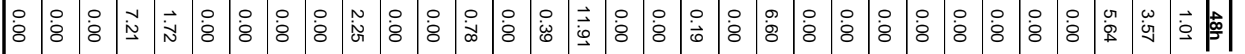

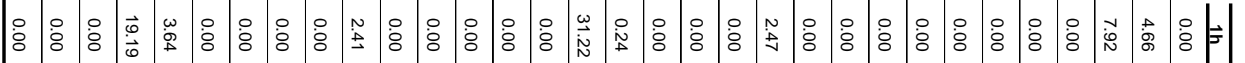

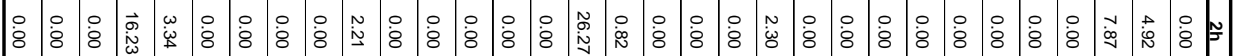

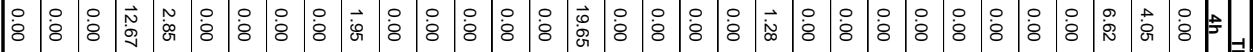

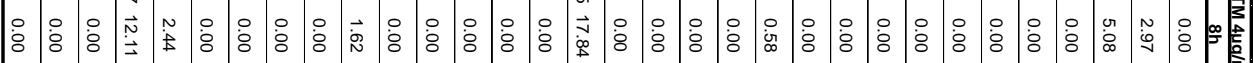

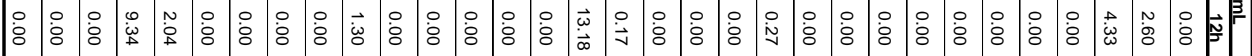

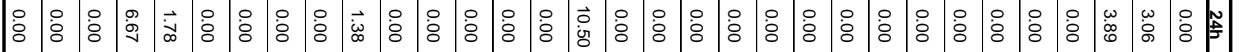

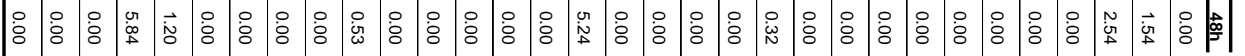

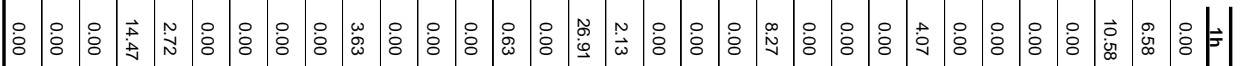

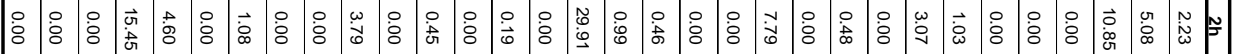

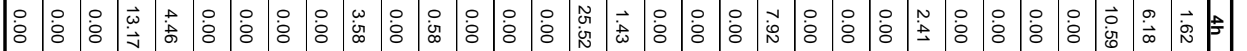

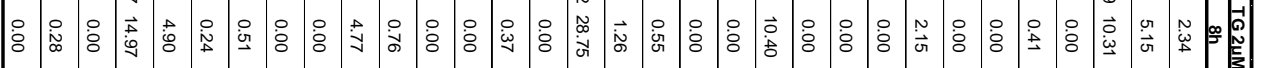

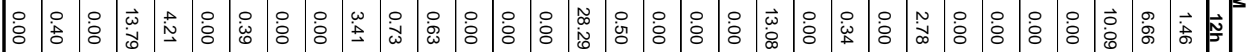

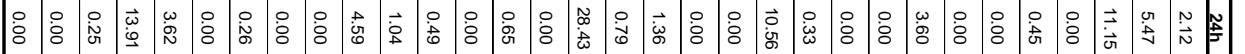

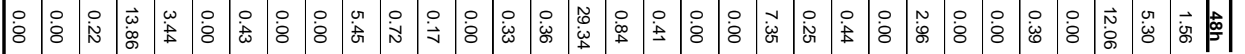

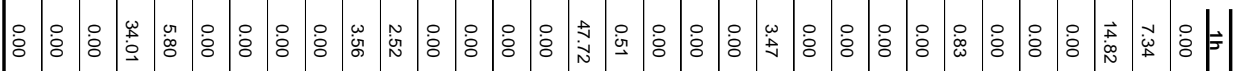

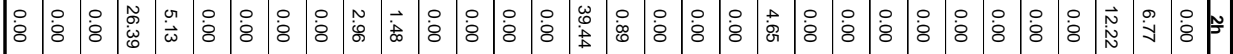

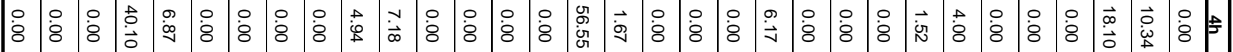

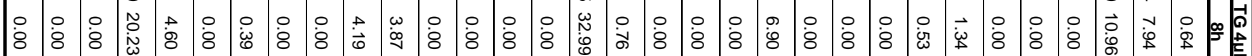

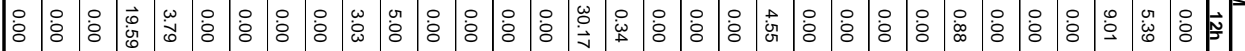

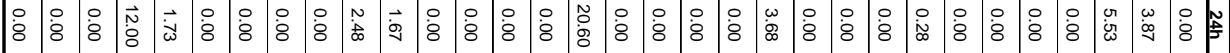

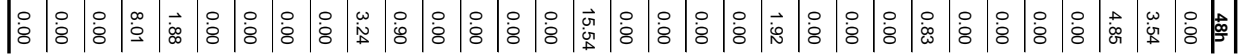




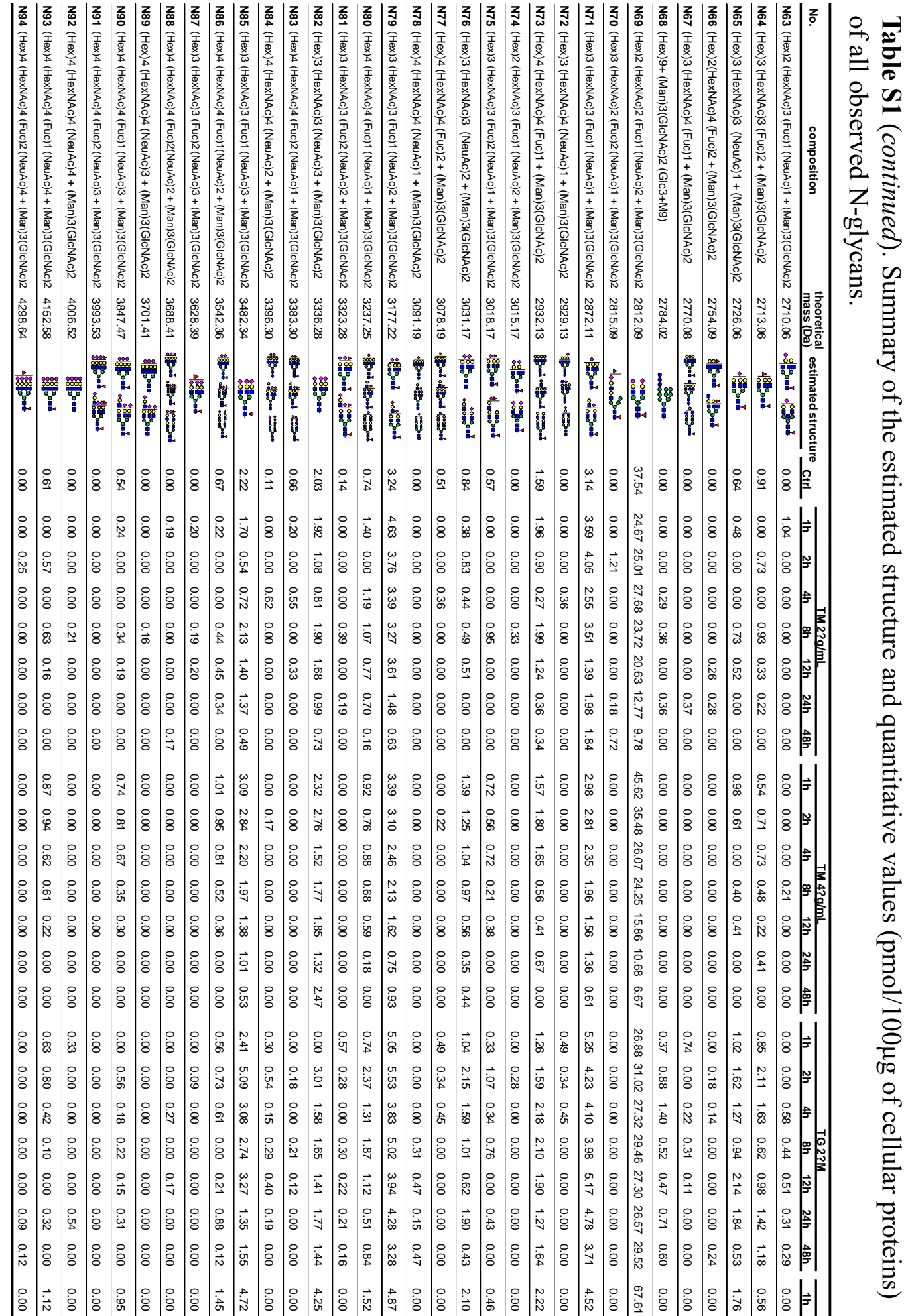

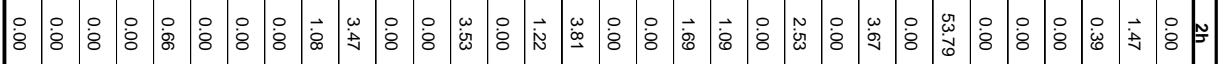

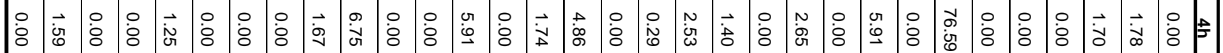

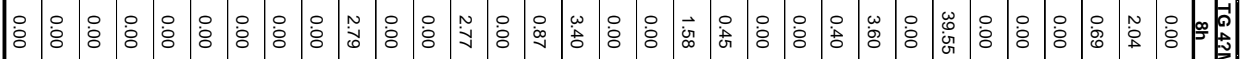

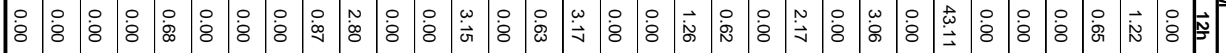

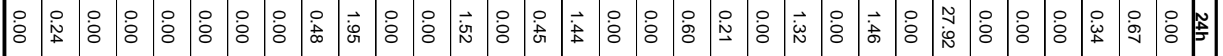

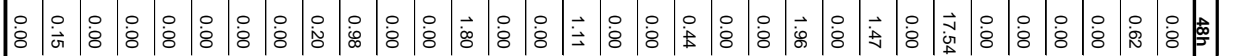




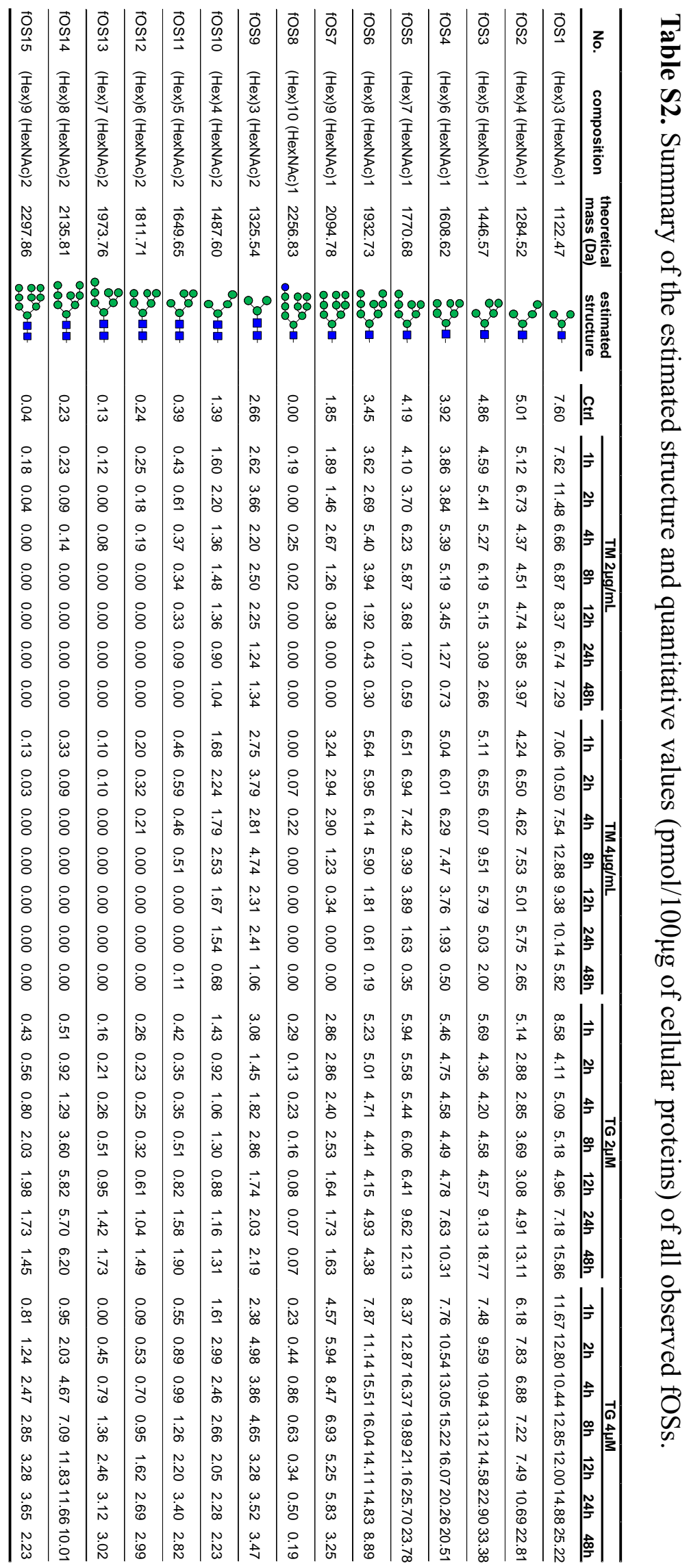



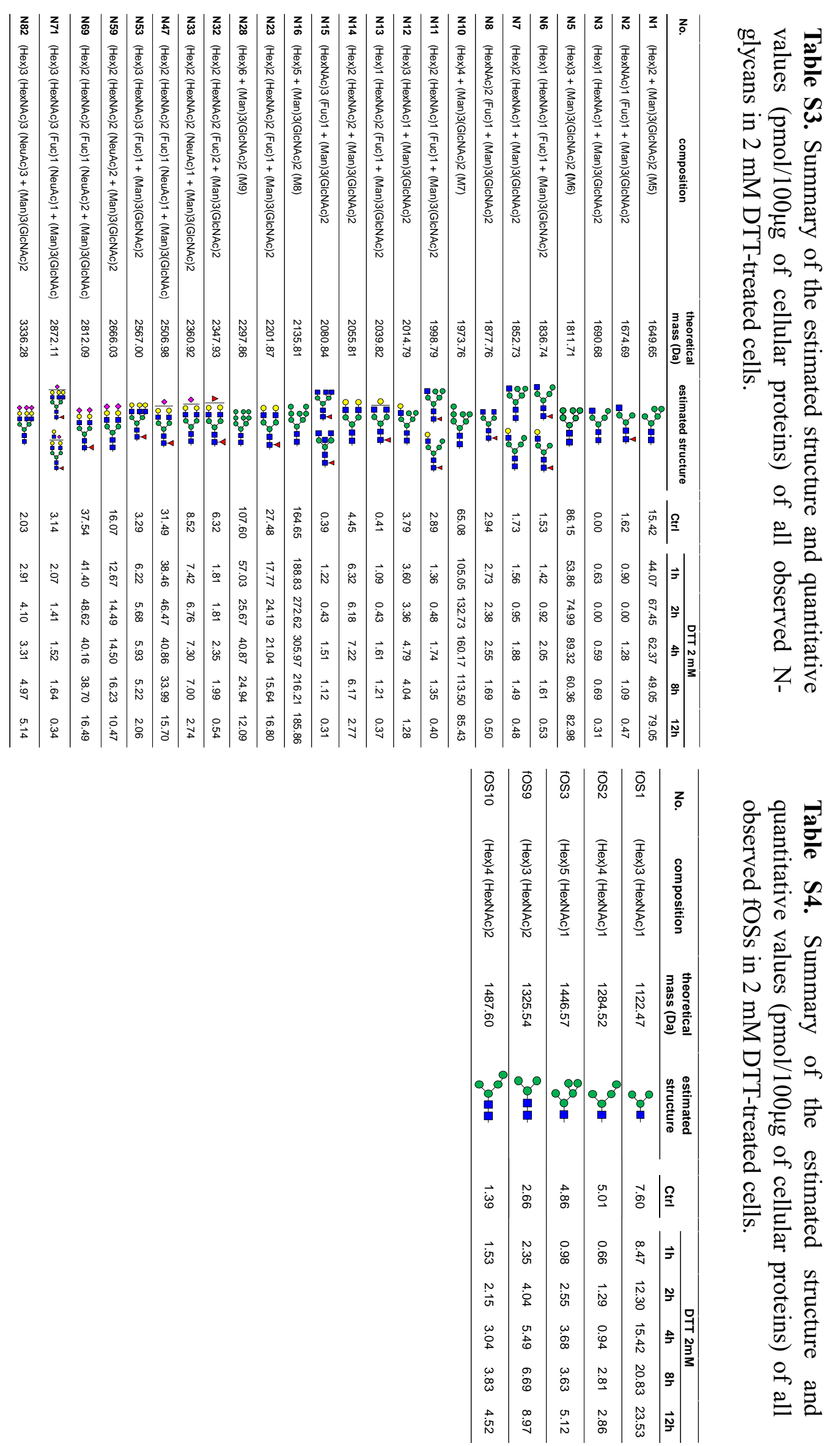